\title{
ANÁLISIS DE LA INVESTIGACIÓN SOBRE CREATIVIDAD EN PUBLICIDAD EN ESPAÑA DE 1980 A 2012
}

\author{
Jorge Del-Río-Pérez y Pablo Medina-Aguerrebere
}

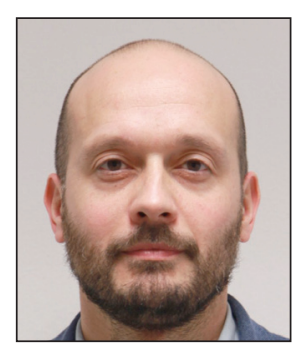

Jorge Del-Río-Pérez es profesor contratado doctor en la Facultad de Comunicación de la Universidad de Navarra (Unav). Su investigación científica trata sobre la creatividad publicitaria tanto en el ámbito de la docencia como en el de la investigación y ha publicado artículos en revistas nacionales e internacionales. Es coordinador del grado de publicidad y relaciones públicas de la Unav. Es miembro a la Asociación Española de Investigación de la Comunicación y de la Academia de la Publicidad.

http://orcid.org/0000-0001-9017-604X

Universidad de Navarra, Dpto. Empresa Informativa Edif. Bibliotecas. Campus Universitario, 31009 Pamplona (Navarra), España jrio@unav.es

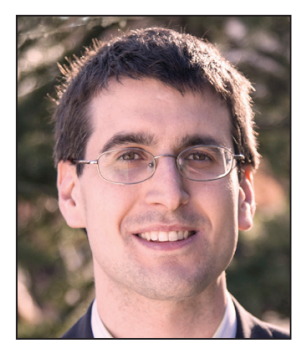

Pablo Medina-Aguerrebere es doctor en comunicación institucional por la Universidad de Navarra, 2011). Ha publicado artículos científicos, libros y comunicaciones de congresos sobre comunicación institucional. Es profesor contratado doctor en la Facultad de Ciencias de la Comunicación de la Universitat Internacional de Catalunya, donde investiga sobre comunicación institucional. http://orcid.org/0000-0003-3101-7035

Universidad Internacional de Cataluña, Facultad de Ciencias de la Comunicación Inmaculada, 22. 08017 Barcelona, España pmedina@uic.es

\section{Resumen}

Se analizan las investigaciones sobre el fenómeno de la creatividad publicitaria publicadas en las revistas científicas españolas de comunicación situadas en el primer y segundo cuartil del índice In-Recs (edición 2011): Anàlisi (de 1980 a 2012), Comunicar (de 1993 a 2012), Comunicación y sociedad (de 1988 a 2012), Doxa (de 2003 a 2012), Questiones publicitarias (de 1993 a 2012), Revista latina de comunicación social (de 1998 a 2012), Trípodos (de 1996 hasta 2012), y Zer (de 1996 a 2012). Mediante el análisis de 3.406 artículos se ha intentado conocer el interés que despierta el estudio de la creatividad publicitaria en el campo de la comunicación. Los resultados revelan que tan sólo el $0,20 \%$ de las investigaciones analizadas abordan el fenómeno de la creatividad publicitaria.

\section{Palabras clave}

Publicidad, Creatividad, Divulgación científica, Revistas científicas, Análisis de contenido.

\section{Title: Analysis of research on creativity in advertising in Spain from 1980-2012}

\begin{abstract}
We provide a content analysis of the research published on advertising creativity in Spanish communication journals of the first and second quartile of the In-Recs index (2011 edition): Anàlisi (from 1980 to 2012), Comunicar (from 1993 to 2012), Comunicación y sociedad (from 1988 to 2012), Doxa (from 2003 to 2012), Questiones publicitarias (from 1993 to 2012), Revista latina de comunicación social(from 1998 to 2012), Trípodos (from 1996 to 2012), and Zer (from 1996 to 2012 ). The authors analysed 3406 papers to determine the level of interest in advertising creativity evidenced in these scientific journals. Only $0.20 \%$ of the studies analyzed focused on advertising creativity.
\end{abstract}

\section{Keywords}

Advertising, Creativity, Research on advertising creativity, Scientific divulgation, Scientific journals, Content analysis.

Del-Río-Pérez, Jorge; Medina-Aguerrebere, Pablo (2014). "Análisis de la investigación sobre creatividad en publicidad en España de 1980 a 2012". El profesional de la información, julio-agosto, v. 23, n. 4, pp. 401-408. 


\section{Introducción}

George Zinkhan, en el editorial del Journal of advertising publicado en 1993, denunció el vacío científico existente en el estudio de la creatividad publicitaria. Tras analizar los artículos publicados en dicha revista, Zinkhan observó que durante los cinco primeros años de publicación, el 10\% de los artículos trataba sobre la creatividad; y, en cambio, durante los siguientes 15 años, ese porcentaje bajó al 1\%. A día de hoy, en el mundo académico anglosajón la creatividad sigue sin generar un gran interés. Así, a modo de ejemplo, se puede señalar que entre 1993 y 2005, de los 984 artículos publicados en las tres principales revistas especializadas en publicidad (Journal of advertising, Journal of advertising research y Journal of current issues and research in advertising), tan sólo 13 abordaron el fenómeno de la creatividad (Del-Río-Pérez, 2006a). Ante esta situación, diversos autores, entre ellos Sasser y Koslow (2008), se han fijado por objetivo la promoción de la investigación sobre este tema, para constituir un marco bien definido de investigación académica sobre creatividad publicitaria.

A pesar de que constituye uno de los elementos fundamentales de la publicidad, la creatividad publicitaria sigue sin interesar al mundo académico

El escaso interés que genera la creatividad publicitaria en el mundo científico anglosajón anima a reflexionar sobre el estado de la cuestión en otros países. Considerando la situación dual existente en el mercado anglosajón, es decir, la gran importancia concedida a la creatividad por parte del sector profesional, y el escaso interés generado por ésta en el mundo académico, este artículo pretende examinar la situación de la investigación sobre creatividad publicitaria en las principales revistas científicas de España. Algunas de las preguntas que nos planteamos son: ¿cuál es el volumen de producción de artículos sobre publicidad en las principales revistas españolas de comunicación? De la bibliografía sobre publicidad, ¿cuál es el número de artículos que se centran en la creatividad publicitaria? Y, finalmente, ¿̇desde qué perspectivas se han acercado los investigadores al fenómeno de la creatividad publicitaria? Para intentar responder a estas preguntas, el artículo reflexiona, en primer lugar, sobre la creatividad publicitaria; en segundo lugar, sobre la investigación académica española centrada en la creatividad publicitaria; en tercer lugar, expone los resultados obtenidos con la investigación empírica; y, en cuarto lugar, ofrece una reflexión a modo de discusión y conclusión.

\section{Creatividad publicitaria}

La creatividad es uno de los elementos centrales del negocio publicitario y constituye una de las vías más efectivas para establecer una comunicación eficaz (Del-Río-Pérez, 2006b). La creatividad presenta los productos o servicios de un modo "alegre, fresco y de forma memorable y persuasiva", lo cual ayuda considerablemente a las marcas a establecer relaciones satisfactorias con sus consumidores (Kuperman, 2000, p. 50). La importancia de la creatividad se plasma, entre otras realidades, en la creación de agencias alrededor del genio creativo de una sola persona (Klebba; Tierney, 1995; Cummings, 1984), como es el caso de Marcel Bleustein-Blanchet (fundador de Publicis), Leo Burnett (fundador de la agencia que lleva su nombre) o George Battent (uno de los fundadores de $B B D O$ ). Entre los académicos y los profesionales existe un consenso sobre el carácter estratégico de la creatividad publicitaria (Roca-Correa; MensaTorras, 2009), la cual se ha convertido en un "componente muy importante de la publicidad" (Till; Baack, 2005, p. 47). Sasser y Koslow (2008, p. 15) insisten en la importancia de la creatividad en una época donde "todo es posible de forma interactiva, con inimaginables opciones ofrecidas por los nuevos medios de comunicación que potencian el diálogo y las relaciones". Esta idea también la recoge Kolster (2012, p. 11): "Es hora de que potenciemos nuestra sabiduría colectiva y nuestra creatividad para llegar a mejores soluciones para el futuro". Por su parte, según la agencia holandesa Kesselskramer (2012), la creatividad publicitaria debe ayudar a las empresas a transmitir sus mensajes de un modo distinto y diferenciador para, de este modo, llegar al consumidor, movilizarle, hacerle pensar y entretenerle.

A pesar de la importancia que tiene la creatividad publicitaria en el sector profesional, la academia no acaba de interesarse por este tema como área de investigación científica. Esto queda demostrado con la investigación realizada por Del-Río-Pérez y Medina-Aguerrebere (2014), quienes tras analizar 4.261 artículos de:

- Current issues and research in advertising (1978 a 2012);

- Journal of advertising (1972 a 2012);

- Journal of advertising research (1960 a 2012);

- International journal of advertising (1990 a 2012),

descubrieron que sólo el 1,19\% de los artículos analizados trataba sobre el fenómeno de la creatividad.

No resulta sencillo identificar las razones que justifiquen el escaso interés académico generado por este tema. Klebba y Tierney (1995) consideran que algunas de ellas tienen que ver con:

- la dificultad para definir el término (Mumford; Gustafson, 1987);

-la propia naturaleza del acto creativo (VanGundy, 1987);

-la diversidad de los productos creativos (Hocevar; Bachelor, 1989);

-el entorno complejo que rodea a la creatividad publicitaria.

Uno de los mayores obstáculos que encuentran los académi-

http://www.tripodos.com 
cos es la definición del concepto "creatividad publicitaria" (Zinkhan, 1993). Esta situación ha provocado que muchos profesionales y académicos consideren que las ideas surgen "por magia", y por tanto, que asocien la creatividad con el "reino del misterio" (Reid; Moriarty, 1983, p. 119). Sin embargo, otros autores han intentado definir dicho concepto. Entre ellos, destacan Reid, King y DeLorme (1998, p. 3), quienes definen la creatividad publicitaria como "el pensamiento original y creativo utilizado para alcanzar objetivos y solucionar problemas publicitarios y comerciales". Los autores sostienen que la creatividad publicitaria constituye una forma especial de creatividad: al contrario que otro tipo de creatividades, la publicitaria se orienta a alcanzar objetivos y solucionar problemas de comunicación (1998). El-Murad y West (2004) resaltan la idea de los anteriores autores y reafirman que la creatividad publicitaria es un tipo de creatividad dirigida hacia la consecución de objetivos concretos: si estos no se logran, la creatividad se considera como un fracaso.

\section{La investigación científica sobre creatividad publicitaria en España}

El desarrollo de la comunicación como ámbito de investigación científica (De-Filippo, 2013) ha propiciado el aumento del número de artículos publicados sobre creatividad publicitaria, que se agrupan en cuatro líneas principales de trabajo (Klebba; Tierney, 1995; Reid; Whitehill; Delorme, 1998; Hackely; Kover, 2007):
a) la persona creativa;
b) el producto creativo;
c) el proceso creativo;
d) el entorno creativo y su influencia en el desarrollo de las ideas.

\section{Sólo el 0,20\% de los artículos analizados trata sobre la creatividad publicitaria}

De las cuatro líneas señaladas, la comunidad académica española se ha centrado especialmente en dos: la persona creativa y el entorno creativo.

En primer lugar, la persona creativa. En esta línea destacan las investigaciones de José-Luis De-León (1999, p. 161), que intenta desentrañar los valores que predominan en los creativos publicitarios y evaluar hasta qué punto "esos valores se trasladan a sus obras, los anuncios, qué percepciones implícitas existen del destinatario, el consumidor, y en qué medida se ven mediatizados por el sistema del management, a cuyo servicio al fin y al cabo se encuentran". Según

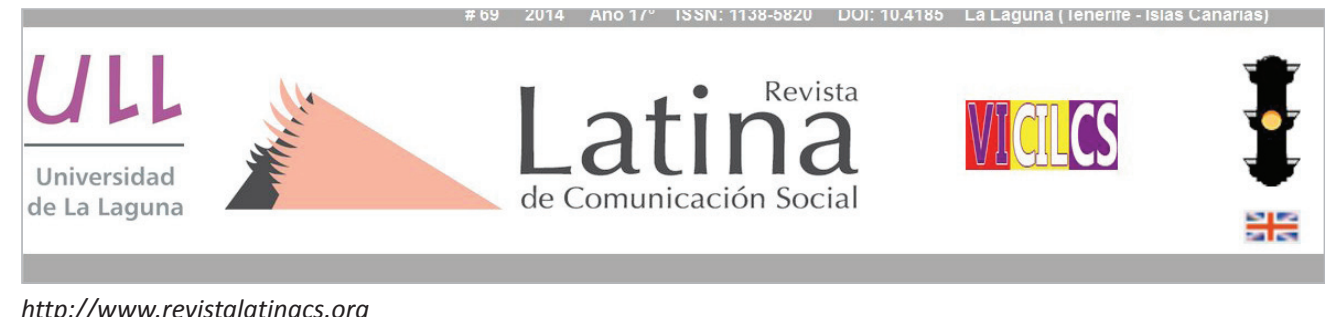

Muchas agencias se constituyen alrededor del genio creativo de una persona, como Marcel Bleustein-Blanchet (Publicis) o George Battent (BBDO)

Por su parte, Mensa-Torras (2012), basando su trabajo en la realización de entrevistas en profundidad con creativos publicitarios, considera que las cualidades personales que mejoran la generación de ideas publicitarias son la observación, la curiosidad, la perseverancia y el inconformismo.

En segundo lugar, el entorno creativo. Sobre este tema destacan las investigaciones que bajo un enfoque eminentemente práctico analizan las variables del entorno profesional que influyen en la creatividad final lograda por la agencia (Del-Río-Pérez, 2006b). Entre dichos factores del entorno, y según Hernández-Martínez (1998), destaca el conocimiento que tiene el creativo sobre el consumidor, de ahí la importancia de investigar sus hábitos de vida y sus cambios de comportamiento. Por su parte, Etayo-Pérez y Del-Río-Pérez (2008b) centran sus investigaciones sobre el entorno creativo, en el papel del director creativo así como en factores relativos a la configuración de los equipos creativos, las técnicas de motivación y la gestión de conflictos. Según los autores, otros de los factores que influyen en la creatividad son, por un lado, la gestión del tiempo de trabajo y, por otro, la difusión de información interna dentro de la agencia que

http://www.revistalatinacs.org 


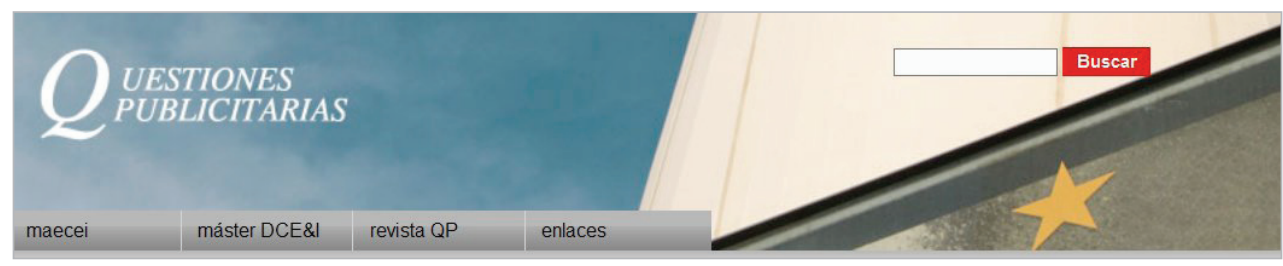

http://www.maecei.es/questiones.html

facilite al creativo estar en contacto constante con el consumidor (Etayo-Pérez; Del-Río-Pérez, 2008a).

Además de las cuatro líneas de trabajo señaladas (persona creativa, producto creativo, proceso creativo y entorno creativo), se pueden indicar otros trabajos que también analizan la creatividad publicitaria. Destacan los investigadores Roca-Correa y Mensa-Torras (2009), quienes centran su trabajo en los instrumentos metodológicos utilizados en los artículos publicados sobre creatividad publicitaria. Según estos autores, los académicos prefieren las investigaciones de tipo empírico $(69,4 \%)$ y no tanto las teóricas $(30,6 \%)$; y dentro de las primeras se opta por lo cuantitativo y no por lo cualitativo: "al menos un $55,5 \%$ de los artículos utilizan esa metodología” (2009, p. 28). Por último, Del-Río-Pérez (2006b) clasifica en cinco grupos las investigaciones académicas realizadas sobre creatividad publicitaria:

a) personalidad del creativo publicitario;

b) producto creativo;

c) proceso creativo;

d) ideación;

e) enfoque multidisciplinar de la creatividad.

\section{Metodología}

La muestra potencial de revistas científicas se seleccionó siguiendo los siguientes criterios:

a) revistas españolas científicas de comunicación situadas en el primer y segundo cuartil del índice In-Recs (edición 2011);

b) revistas con más de 10 años de publicación;

c) revistas que contuvieran la categoría de "publicidad".

De la muestra resultante se eliminó la publicación Estudios sobre el mensaje periodístico porque no respondía a este último criterio. Finalmente, el trabajo empírico se centró en las siguientes revistas:

- Anàlisi (1980 a 2012)

- Comunicar (1993 a 2012)

- Comunicación y sociedad (C\&S) (1988 a 2012)

- Doxa (2003 a 2012)

- Revista latina de comunicación social (RLCS) (1998 a 2012)

- Questiones publicitarias (QP) (1993 a 2012)

- Trípodos (1996 a 2012)

- Zer (1996 a 2012) el entorno creativo

\section{Resultados}

Para cada revista se tomaron en consideración los índices de todos y cada uno de los números publicados para así poder identificar artículos que, en principio, tratasen sobre el fenómeno de la publicidad y de la creatividad. Para el análisis de cada revista los términos de búsqueda utilizados -en el título, resumen y palabras clave- fueron: "creatividad", "creativo", "creatividad publicitaria", "creación”, "ideación” y "publicidad”. A continuación, un análisis de contenido determinó si el artículo identificado versaba o no sobre los elementos de búsqueda. Se puso especial atención en que los artículos tuviesen suficiente identidad científica para ser incluidos en la muestra. Así, por ejemplo, los editoriales, revisiones de libros y entrevistas no fueron considerados ya que no se trataban de artículos de investigación. En total se revisaron 3.406 artículos.

Los estudios de creatividad se agrupan en cuatro líneas: la persona creativa, el producto creativo, el proceso creativo y

Los resultados hallados se exponen en tres grandes bloques:

a) artículos publicados sobre publicidad;

b) artículos publicados sobre creatividad;

c) artículos publicados sobre creatividad publicitaria en comparación con los artículos publicados sobre publicidad.

Se ha aplicado este orden deductivo para así ilustrar de un modo claro cuál es el verdadero interés que genera la creatividad dentro de la investigación científica sobre publicidad.

En lo que se refiere al primer bloque de resultados, y siguiendo los datos aportados por la tabla 1, se puede afirmar que la publicidad no es un asunto relevante dentro de los trabajos académicos sobre comunicación. Así, de los 3.406 artículos identificados, 225 tratan sobre publicidad $(6,60 \%$ de los artículos analizados. Entre todas las revistas tratadas, destaca el caso de Questiones publicitarias, cuyo porcentaje de artículos dedicados a la publicidad es del 76,22\%.

El segundo bloque de resultados, centrado en los artículos dedicados a la creatividad publicitaria, pone en evidencia la falta de interés de la comunidad académica hacia este tema. En la tabla 2 se puede observar que, sobre el total de los 3.406 artículos publicados, sólo 7 están centrados en la creatividad. Los datos obtenidos demuestran que las mejores revistas españolas de comunicación manifiestan, al igual que sucede en el mundo anglosajón, un insuficiente interés hacia la creatividad. Entre todas ellas, destaca positivamente el caso de Zer, 
Tabla 1. Artículos publicados sobre publicidad

\begin{tabular}{|l|c|c|c|}
\hline \multicolumn{1}{|c|}{ Revistas } & $\begin{array}{c}\text { Artículos publi- } \\
\text { cidad }\end{array}$ & Total artículos & $\%$ \\
\hline Anàlisi & 5 & 219 & 2,28 \\
\hline Comunicar & 14 & 916 & 1,52 \\
\hline C\&S & 19 & 315 & 6,03 \\
\hline Doxa & 10 & 136 & 7,35 \\
\hline QP & 93 & 122 & 76,22 \\
\hline RLCS & 32 & 936 & 3,41 \\
\hline Trípodos & 26 & 329 & 7,90 \\
\hline ZER & 26 & 433 & 6,00 \\
\hline Total & 225 & 3.406 & 6,60 \\
\hline
\end{tabular}

que ha publicado un total de 4 artículos sobre el fenómeno de la creatividad. A continuación, y con 2 artículos menos, aparece Comunicación y sociedad. Por otro lado, también se puede señalar el caso de Anàlisi, Doxa, Revista latina de comunicación social, Trípodos y Comunicar, que no presentan en sus volúmenes ninguna investigación sobre este área. EI negocio de la publicidad, como señalan Till y Baack (2005), se sustenta sobre la palabra y el concepto de creatividad. Sin embargo, la realidad nos revela que la comunidad académica no se interesa lo suficiente por la creatividad: según los datos aportados, sólo el $0,20 \%$ de los artículos analizados trata sobre este fenómeno. La situación académica en España es similar a lo que sucede a nivel internacional donde, como se ha señalado anteriormente, sólo el $1,19 \%$ de los artículos publicados en cuatro prestigiosas revistas internacionales de publicidad tratan el fenómeno de la creatividad. Se trata de un porcentaje muy bajo, especialmente si lo comparamos con el estudio realizado por Zou (2005), del que se desprende que, entre 1990 y 2002, el 12,20\% de los artículos publicados en tres grandes revistas (Journal of advertising, Journal of advertising research y Journal of current issues and research in advertising) trataba sobre el fenómeno del international advertising, uno de los temas más candentes en el sector de la publicidad en aquellos años.

En estos últimos años se observa un cambio de tendencia que se plasma en un aumento en el número de publicaciones sobre creatividad

En cuanto al tercer bloque de resultados, y siguiendo los datos de la tabla 3 , se puede resaltar que el 3,11\% de los artículos que aborda el área de la publicidad se centra en el fenómeno de la creatividad. Destacan los casos de Zer y de Comunicación y sociedad, cuyos porcentajes de artículos sobre creatividad en comparación con los publicados sobre publicidad superan el $10 \%$ (15,38\% y $10,52 \%$ respectivamente). Esta realidad, que se da tanto en la comunidad científica española como en la anglosajona, demuestra que en los próximos años será necesario que los académicos de dichos países concedan una importancia creciente a la investigación sobre creatividad.
Tabla 2. Artículos publicados sobre creatividad publicitaria

\begin{tabular}{|l|c|c|c|}
\hline \multicolumn{1}{|c|}{ Revistas } & $\begin{array}{c}\text { Artículos creativi- } \\
\text { dad publicitaria }\end{array}$ & Total artículos & $\%$ \\
\hline Anàlisi & 0 & 219 & 0 \\
\hline Comunicar & 0 & 916 & 0 \\
\hline C\&S & 2 & 315 & 0,63 \\
\hline Doxa & 0 & 136 & 0 \\
\hline RLCS & 0 & 936 & 0 \\
\hline QP & 1 & 122 & 0,81 \\
\hline Trípodos & 0 & 329 & 0 \\
\hline Zer & 4 & 433 & 0,92 \\
\hline Total & 7 & 3.406 & 0,20 \\
\hline
\end{tabular}

En cuanto a la productividad de los investigadores expertos en publicidad, destacan los autores Rey-Fuentes, RocaCorrea y Sabaté-López (tabla 4). Para establecer este ranking de producción científica por investigador, se siguió el criterio utilizado por Zou (2005). Así, de los 225 artículos, se contabilizaron las contribuciones individuales con un punto; 0,5, si el artículo lo firmaban dos autores; y 0,3, si el texto lo habían elaborado entre tres investigadores.

\section{Discusión y conclusiones}

A pesar de que la comunicación como disciplina se está consolidando en España como área científica (Fernández; Masip, 2013; De-Filippo, 2013), la comunidad académica española no acaba de interesarse del todo por la publicidad como área de investigación. Esto queda demostrado con un dato: de los 3.406 artículos analizados en este estudio, sólo 225 tratan sobre publicidad. También hay que señalar que en todas las revistas consideradas, salvo Questiones publicitarias, Doxa y Trípodos, los artículos sobre publicidad no representan más del 6,03\% del total de artículos publicados. Esta falta de interés por la publicidad también se plasma, entre otras realidades, en el número de artículos tan reducido que dichas revistas dedican la creatividad publicitaria. De todas las revistas consideradas en este estudio, un total de cinco (Anàlisi, Comunicar, Doxa, Revista latina de comunicación social, y Trípodos) no han publicado nada sobre este tema. Y, el resto de las revistas sólo han publicado 7 artículos: Zer (4), Comunicación y sociedad (2) y Questiones publicitarias (1).

Tabla 3. Artículos sobre creatividad publicitaria vs. artículos sobre publicidad

\begin{tabular}{|l|c|c|c|}
\hline Revistas & $\begin{array}{c}\text { Artículos creativi- } \\
\text { dad publicitaria }\end{array}$ & $\begin{array}{c}\text { Artículos } \\
\text { publicidad }\end{array}$ & $\%$ \\
\hline Anàlisi & 0 & 5 & 0 \\
\hline Comunicar & 0 & 14 & 0 \\
\hline C\&S & 2 & 19 & 10,52 \\
\hline Doxa & 0 & 10 & 0 \\
\hline RLCS & 0 & 32 & 0 \\
\hline QP & 1 & 93 & 1,07 \\
\hline Trípodos & 0 & 26 & 0 \\
\hline Zer & 4 & 26 & 15,38 \\
\hline Total & 7 & 225 & 3,11 \\
\hline
\end{tabular}


Tabla 4. Ranking de autores con mayor número de publicaciones sobre publicidad

\begin{tabular}{|l|c|}
\hline \multicolumn{1}{|c|}{ Autor } & $\begin{array}{c}\text { Producción } \\
\text { científica }\end{array}$ \\
\hline Rey-Fuentes, Juan & 9,50 \\
\hline Roca-Correa, David; Sabaté-López, Joan & 4,50 \\
\hline Garrido-Lora, Manuel & 4,00 \\
\hline Sánchez-Blanco, Cristina & 3,50 \\
\hline Muela-Molina, Clara; Papí-Gálvez, Natalia; Pérez-Latre, Francisco-Javier; Rodríguez-Rad, Carlos J. & 3,00 \\
\hline Baladrón-Pazos, Antonio; Fanjul-Peyró, Carlos; González-Oñate, Cristina & 2,50 \\
\hline Martín-Llaguno, Marta & 2,33 \\
\hline $\begin{array}{l}\text { Alonso-González, Carmen-María; Cortés-González, Alfonso; Del-Río-Pérez, Jorge; Fernández-Poyatos, María-Dolores; Huici, Adrián; } \\
\text { Méndiz-Noguero, Alfonso; Montoya-Vilar, Norminanda; Rodríguez-Centeno, Juan-Carlos; Vergara-Leyton, Enrique }\end{array}$ & 2,00 \\
\hline $\begin{array}{l}\text { Bringué-Sala, Xavier; Etayo-Pérez, Cristina; Fernández-Gómez, Jorge-David; García-Gónzalez, Aurora; Ruiz-Granados, Pilar-Lara; } \\
\text { Lasso-de-la-Vega, Carmen; López-Font, Lorena; Mensa-Torras, Marta; Piñeiro-Otero, Teresa; Quintana-Daza, Miguel-Ángel; Rom- } \\
\text { Rodríguez, Josep A.; Sorribas-Morales, Carolina }\end{array}$ & 1,50 \\
\hline Curto, Víctor; Hernández-Ruiz, Alejandra & 1,33 \\
\hline
\end{tabular}

revista de estudios de comunicación

komunikazio ikasketen aldizkaria

journal of communication studies

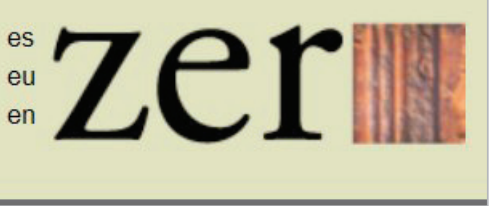

http://www.ehu.es/zer

La creatividad al servicio del cliente es el principal producto que ofrece una agencia de publicidad (Bilton, 2007, p. 30), sin embargo, el carácter estratégico de este área no acaba de implantarse en el mundo académico, donde sólo unos pocos autores, especialmente Rey, Roca-Correa, Sabaté y GarridoLora, dedican sus esfuerzos a investigar sobre creatividad publicitaria. El esfuerzo de estos autores puede ayudar a aumentar el número de artículos sobre publicidad que se centran en la creatividad, los cuales, según el estudio, tan sólo representan el 3,11\% (es decir, de los 225 artículos identificados sobre publicidad, sólo 7 se centran en la creatividad). En el mundo académico, faltan investigaciones que permitan construir un "paradigma científico multidisciplinar que sostenga el estudio cualitativo de la creatividad publicitaria mediante el desarrollo de metodologías apropiadas para ello" (NicolásOjedo; Miralles; Grandío-Pérez, 2011, p. 8). La investigación sobre este ámbito "tiene una segunda oportunidad para abordar trabajos con enfoques pertinentes que ayuden a dar respuestas a las cuestiones de fondo sobre este fenómeno" (Del-Río-Pérez; Sánchez-Blanco, 2011, p. 66). Pero, para ello, resulta clave, como señalan Nicolás-Ojedo, Miralles y Grandío-Pérez (2011, p. 8), que la comunidad académica se centre en fijar "líneas de investigación sobre creatividad publicitaria bien definidas y metodologías que no dejen de observar y tener presente la práctica profesional". taciones de este artículo:

\section{Bibliografía} 115-130. Publishing. ISBN: 1405119969

etc.), se pueden señalar

- la complejidad a la hora de definir el concepto de "creatividad publicitaria" ha dificultado la creación de un marco investigador que facilite la integración de académicos de varios países expertos en este tema, lo cual explica en parte el número tan escaso de artículos publicados sobre creatividad publicitaria;

- los investigadores sobre creatividad deben recurrir a técnicas cuantitativas que permitan reflejar con datos empíricos las realidades profesionales vividas en las agencias, donde la mayor parte de los empleados asumen el rol tan importante de la creatividad publicitaria, lo cual ayudará a la comunidad académica a comprender la importancia estratégica de este área de investigación.

Ashley, Christy; Oliver, Jason (2010). “Creative leaders. Thirty years of big ideas". Journal of advertising, v. 39, n. 1, pp.

Bilton, Chris (2007). Management and creativity: from creative industries to creative management. Oxford: Clackwell

Cummings, Bart (1984). The benevolent dictators: interviews with the advertising greats. Chicago: Grain Publishing. ISBN: 0844231916.

ENGLISH VERSION

\section{COMUNICACIÓN Y SOCIEDAD}

Universidad de Navarra Facultad de Comunicación
De-Filippo, Daniela (2013). "Spanish scientific output in communication sciences in WoS. The scientific journals in SSCl (2007$12)$ ". Comunicar, n. 41, pp. 25-34.

http://www.unav.es/fcom/comunicacionysociedad/es 
http://www.revistacomunicar. com/index.php?contenido $=d$ etalles\&numero $=41 \&$ articu lo $=41-2013-02$

http://dx.doi.org/10.3916/ C41-2013-02

De-León, José-Luis (1999). "Creativos publicitarios: una visión del mundo". Zer, v. 7, n. 4, pp.

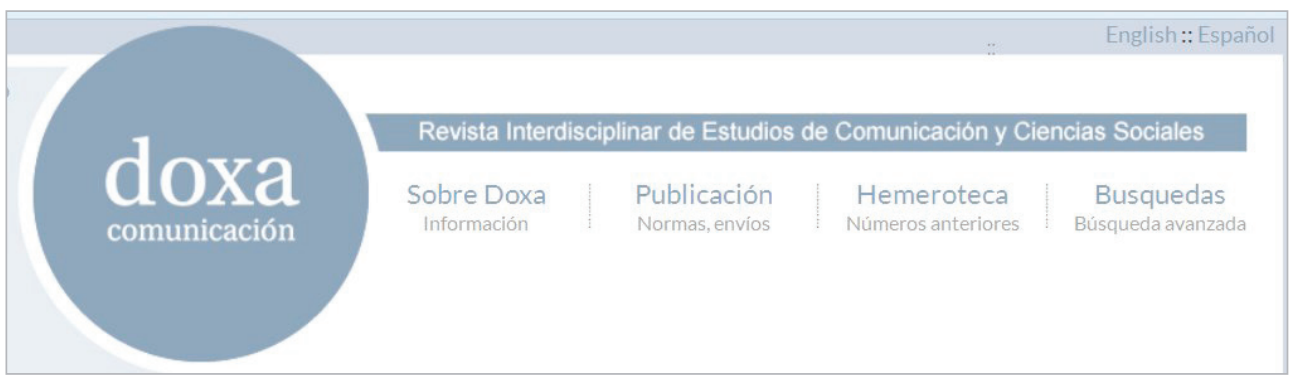

http://www.doxacomunicacion.es 161-179.

http://www.ehu.es/zer/hemeroteca/pdfs/zer07-09-deleon.pdf

Del-Río-Pérez, Jorge (2006a). “Contribuciones a la investigación sobre la creatividad publicitaria. Una valoración de la literatura publicada entre 1965 y 2005". Trípodos, v. extra, pp. 31-39.

http://dspace.unav.es/dspace/handle/10171/21355

Del-Río-Pérez, Jorge (2006b). “La creatividad publicitaria en la literatura científica: una revisión”. Comunicación y sociedad, v. 19, n. 1, pp. 9-46.

http://www.unav.es/fcom/comunicacionysociedad/es/ articulo.php?art_id $=54$

Del-Río-Pérez, Jorge; Medina-Aguerrebere, Pablo (2014). "La diffusion scientifique de la créativité publicitaire de 1965 à 2012". Communication, v. 38, n. 1, pp. 53-63.

Del-Río-Pérez, Jorge; Sánchez-Blanco, Cristina (2011). “EI futuro de la investigación sobre creatividad publicitaria en el nuevo entorno cultural de la comunicación". Sphera publica, v. 11, pp. 57-68.

http://www.redalyc.org/pdf/297/29729580004.pdf

El-Murad, Jaafar; West, Douglas (2004). "The definition and measurement of creativity: what do we know?". Journal of advertising research, June, v. 44, n. 2, pp. 188-201.

http://dx.doi.org/10.1017/S0021849904040097

Etayo-Pérez, Cristina; Del-Río-Pérez, Jorge (2008a). “Influencia de la organización del tiempo sobre la creatividad en las agencias de publicidad". Questiones publicitarias, v. 1, n. 13, pp. 27-37.

http://dspace.unav.es/dspace/handle/10171/22499

Etayo-Pérez, Cristina; Del-Río-Pérez, Jorge (2008b). “Influencia de los factores humanos sobre la creatividad en las agencias de publicidad". Zer, v. 13, n. 25, pp. 197-219.

http://www.ehu.es/zer/hemeroteca/pdfs/zer25-10-etayo.pdf

Fernández-Quijada, David; Masip-Masip, Pere (2013). "Tres décadas de investigación española en comunicación: hacia la mayoría de edad". Comunicar, v. 41, pp. 15-24. http://www.revistacomunicar.com/index.php?contenido=d etalles\&numero $=41 \&$ articulo $=41-2013-01$

http://dx.doi.org/10.3916/C41-2013-01

Grow, Jean; Roca-Correa, David; Broyle, Sheri (2012). "Vanishing acts. Creative women in Spain and United States". International journal of advertising, v. 31, n. 3, pp. 657-679. http://dx.doi.org/10.2501/IJA-31-3-657-679

Hackley, Chris; Kover, Arthur (2007). "The trouble with creatives: negating creative identity in advertising agencies".

International journal of advertising, v. 26, n. 1, pp. 63-78.

Hernández-Martínez, Caridad (1998). “Creatividad publicitaria y contexto social". Zer, v. 4, n. 3, pp. 239-263.

http://www.ehu.es/zer/hemeroteca/pdfs/zer04-11hernandez.pdf

Hocevar, Dennis; Bachelor, Patricia (1989). "Taxonomy and critique of measurements used in the study of creativity". En: Glover, John; Ronning, Royce; Reynolds, Cecil. Handbook of creativity. New York: Plennum, pp. 53-75. ISBN: 978 1441932129

http://dx.doi.org/10.1007/978-1-4757-5356-1_3

Kesselskramer (2012). Advertising for people who don't like advertising. London: Laurence King Publishing. ISBN: 1780673205

Klebba, Joanne; Tierney, Pamela (1995). "Advertising creativity: a review and empirical investigation of external evaluation, cognitive style and self-perceptions of creativity". Journal of current issues and research in advertising, v. 17, n. 2, pp. 33-52.

http://dx.doi.org/10.1080/10641734.1995.10505031

Kolster, Thomas (2012). Goodvertising. Creative advertising that cares. London: Thames \& Hudson. ISBN: 050051626X

Koslow, Scott; Sasser, Sheila; Riordan, Edward (2006). “Do marketers get the advertising they need or the advertising they deserve? Agency views of how clients influence creativity". Journal of advertising, v. 35, n. 3, pp. 81-101. http://dx.doi.org/10.2753/JOA0091-3367350306

Kuperman, Bob (2000). "Risky business". Adweek, 11 Nov.

Mensa-Torras, Marta (2012). "Creativos publicitarios en Perú: características y entorno laboral". Zer, v. 17, n. 33, pp. 47-66.

http://www.ehu.es/zer/hemeroteca/pdfs/zer33-03-mensa.pdf

Mumford, Michael; Gustafson, Sigrid (1988). "Creativity syndrome: integration, application, and innovation". Psychological bulletin, n. 103, n. 1, pp. 27-43.

http://dx.doi.org/10.1037/0033-2909.103.1.27

Nicolás-Ojedo, Miguel-Ángel; Miralles-González-Conde, María A.; Grandío-Pérez, María-del-Mar (2011). "La disciplina publicitaria y sus ámbitos de transformación". Sphera pública, n. 11, pp. 7-18.

http://www.redalyc.org/pdf/297/29729580001.pdf

Reid, Leonard; Moriarty, Sandra (1983). "Ideation: a review of research". Current issues and research in advertising, v. 6, n. 2, pp. 119-134. 
Reid, Leonard; King, Karen; Delorme, Denise (1998). “Toplevel agency creatives look at advertising creativity then and now". Journal of advertising, v. 27, n. 2, pp. 1-16. http://dx.doi.org/10.1080/00913367.1998.10673549

Roca-Correa, David; Mensa-Torras, Marta (2009). "Las metodologías utilizadas en las investigaciones de creatividad publicitaria (1965-2007)". Comunicación y sociedad, v. 22, n. 1, pp. 7-34. http://dspace.unaves/dspace/bitstream/10171/8629/1/20091202125417. pdf

Sasser, Sheila; Koslow, Scott (2008). “Desperately seeking advertising creativity. Engaging an imaginative "3Ps» research agenda". Journal of advertising, v. 37, n. 4, pp. 5-20. http://dx.doi.org/10.2753/JOA0091-3367370401

Till, Brian; Baack, Daniel (2005). "Recall and persuasion.
Does creative advertising matter?". Journal of advertising, v. 34, n. 3, pp. 47-57.

http://dx.doi.org/10.1080/00913367.2005.10639201

VanGundy, Arthur (1987). "Organizational creativity and innovation". En: Isaken, S. Frontiers of creativity research: beyond the basis. Buffalo: Bearly Limited, pp. 358-379. ISBN: 9780943456249

Zinkhan, George (1993). "Creativity in advertising: creativity in the Journal of advertising". Journal of advertising, v. 12, n. 3, pp. 1-3.

http://dx.doi.org/10.1080/00913367.1993.10673398

Zou, Shaoming (2005). "Contributions to international advertising research". Journal of advertising, v. 34, n. 1, pp. 99-110. http://dx.doi.org/10.1080/00913367.2005.10639185

\section{Directorio EXIT (EXpertos en Tratamiento de la Información) http://directorioexit.info}

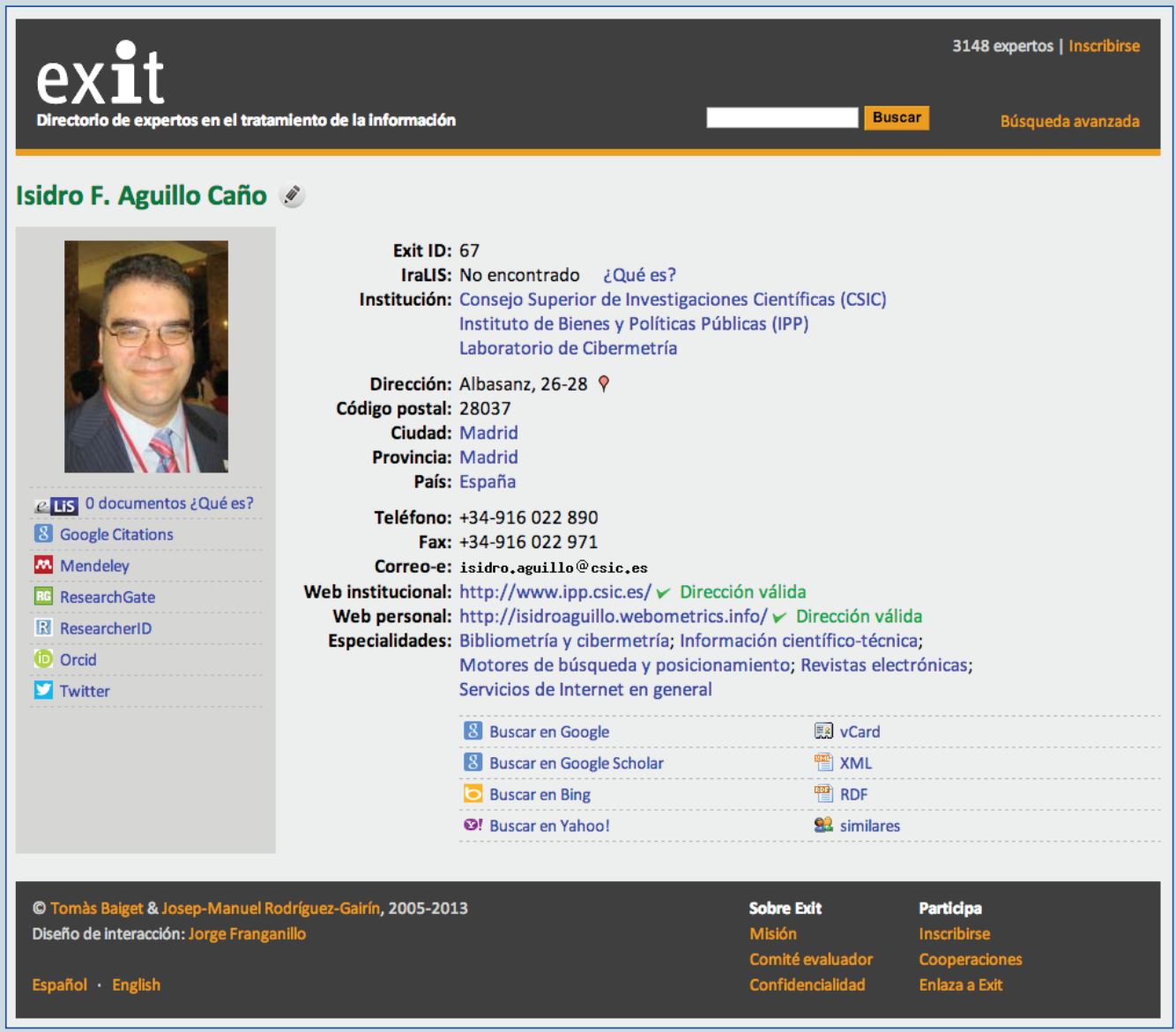

\section{Una ventana a las oportunidades profesionales}

